\title{
Thermal Radiation and Amplified Spontaneous Emission from a Random Medium
}

\author{
C. W. J. Beenakker \\ Instituut-Lorentz, Leiden University, P.O. Box 9506, 2300 RA Leiden, The Netherlands
}

(Received 28 January 1998)

\begin{abstract}
We compute the statistics of thermal emission from systems in which the radiation is scattered chaotically, by relating the photocount distribution to the scattering matrix - whose statistical properties are known from random-matrix theory. We find that the super-Poissonian noise is that of a blackbody with a reduced number of degrees of freedom. The general theory is applied to a disordered slab and to a chaotic cavity, and is extended to include amplifying as well as absorbing systems. We predict an excess noise of amplified spontaneous emission in a random laser below the laser threshold. [S0031-9007(98)07001-X]
\end{abstract}

PACS numbers: 42.50.Ar, 05.45.+b, 42.25.Bs, 78.45.+h

The emission of photons by matter in thermal equilibrium is not a series of independent events. The textbook example is blackbody radiation [1,2]: Consider a system in thermal equilibrium (temperature $T$ ) that fully absorbs any incident radiation in $N(\omega)$ propagating modes within a frequency interval $\delta \omega$ around $\omega$. A photodetector counts the emission of $n$ photons in this frequency interval during a long time $t \gg 1 / \delta \omega$. The probability distribution $P(n)$ is given by the negative-binomial distribution with $\nu=N t \delta \omega / 2 \pi$ degrees of freedom,

$$
P(n) \propto\left(\begin{array}{c}
n+\nu-1 \\
n
\end{array}\right) \exp \left(-n \hbar \omega / k_{\mathrm{B}} T\right) .
$$

The binomial coefficient counts the number of partitions of $n$ bosons among $\nu$ states. The mean photocount $\bar{n}=\nu f$ is proportional to the Bose-Einstein function

$$
f(\omega, T)=\left[\exp \left(\hbar \omega / k_{\mathrm{B}} T\right)-1\right]^{-1} .
$$

In the limit $\bar{n} / \nu \rightarrow 0$, Eq. (1) approaches the Poisson distribution $P(n) \propto \bar{n}^{n} / n$ ! of independent photocounts. The Poisson distribution has variance $\operatorname{Var}(n=\bar{n})$ equal to its mean. The negative-binomial distribution describes photocounts that occur in "bunches," leading to an increase of the variance by a factor $1+\bar{n} / \nu$. These basic facts have been known since the beginning of this century [3].

Thermal radiation is also referred to as "chaotic radiation" $[1,2]$. In recent years the word "chaotic" has entered optics in a different context, to describe systems that scatter radiation in an irregular, random way [4]. Such systems, typically, have weak absorption, so they are far from being blackbodies. Two recent papers have studied deviations from blackbody radiation in the case of one-dimensional scattering [5,6], but chaotic systems are intrinsically not one dimensional. What, then, is the statistics of the chaotic radiation resulting from chaotic scattering? That is the problem addressed in this paper.

This problem is significant for more than one reason. First, thermal emission is a fundamental property of a sys- tem. Deviations from the blackbody limit contain information on chaotic scattering that cannot be obtained from classical scattering experiments. Most studies of the optical properties of random media have been restricted to classical optics [7]. The similarity between the classical wave equation and the Schrödinger equation has permitted the transfer to classical optics of powerful theoretical techniques from condensed matter physics [8]. Our solution of the thermal-radiation problem demonstrates how one of these techniques, the method of random-matrix theory [9], can be applied to quantum optics. That is the second reason for the significance of this problem. The third reason is the recent interest in amplifying random media, motivated by possible applications as a "random laser" [10,11]. A linear amplifier can be thought of as being in thermal equilibrium at a negative temperature [12], so that our theory of thermal radiation can also deal with amplified spontaneous emission.

We start with the formulation and solution of the problem in a general form, and then turn to specific applications. We consider a random medium coupled to a photodetector via a waveguide (in vacuum) with $N(\omega)$ propagating modes (counting polarizations) at frequency $\omega$ (see Fig. 1). We assume that any Brownian motion of the scattering centra in the random medium can be disregarded on the time scale of the measurements. The scattering rate is denoted by $1 / \tau_{\mathrm{s}}$, and the absorption or

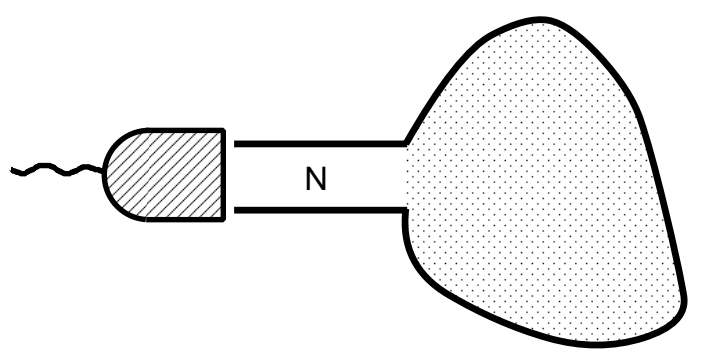

FIG. 1. Schematic diagram of a random medium (dotted) connected to a photodetector (shaded) via an $N$-mode waveguide. 
amplification rate by $1 / \tau_{\mathrm{a}}$. To quantize the electromagnetic field we use the method of input-output relations developed by Gruner and Welsch [5] and by Loudon and co-workers $[6,12,13]$. The incoming and outgoing modes in the waveguide are represented by two $N$-component vectors of annihilation operators $a^{\text {in }}(\omega), a^{\text {out }}(\omega)$. They satisfy the commutation relations

$$
\begin{aligned}
& {\left[a_{n}(\omega), a_{m}^{\dagger}\left(\omega^{\prime}\right)\right]=\delta_{n m} \delta\left(\omega-\omega^{\prime}\right),} \\
& {\left[a_{n}(\omega), a_{m}\left(\omega^{\prime}\right)\right]=0,}
\end{aligned}
$$

for $a=a^{\text {in }}$ or $a=a^{\text {out }}$. The input-output relations take the form $[5,6,12,13]$

$$
a^{\text {out }}=S a^{\text {in }}+U b+V c^{\dagger},
$$

with $S(\omega)$ the $N \times N$ scattering matrix. The boson operators $b$ and $c$ satisfy Eq. (3) provided

$$
U U^{\dagger}-V V^{\dagger}=\mathbb{1}-S S^{\dagger}
$$

( $\mathbb{1}$ denoting the $N \times N$ unit matrix). The matrix $\mathbb{1}-S S^{\dagger}$ is positive definite in an absorbing medium, so we can put $V=0$. Conversely, in an amplifying medium $\mathbb{1}-S S^{\dagger}$ is negative definite, so we can put $U=0$. This determines $U, V$ up to a unitary transformation. All of our final expressions depend only on the combination $U U^{\dagger}-V V^{\dagger}$, so that any freedom in the choice of $U, V$ is irrelevant once the scattering matrix is fixed.

Equation (5) can be understood as a fluctuationdissipation relation: The left-hand side accounts for quantum fluctuations in the electromagnetic field due to spontaneous emission or absorption of photons, and the right-hand side accounts for dissipation due to absorption (or stimulated emission in the case of an amplifying medium). Equation (5) also represents a link between classical optics (the scattering matrix $S$ ) and quantum optics (the quantum fluctuation matrices $U, V$ ).

In an absorbing medium, the operator $b$ accounts for thermal emission with expectation value

$$
\left\langle b_{n}^{\dagger}(\omega) b_{m}\left(\omega^{\prime}\right)\right\rangle=\delta_{n m} \delta\left(\omega-\omega^{\prime}\right) f(\omega, T) .
$$

The inverted oscillator $c$ accounts for spontaneous emission in an amplifying medium. We consider the regime of linear amplification, below the laser threshold. Formally, this regime can be described by a thermal distribution at negative temperature $-T$,

$$
\left\langle c_{n}(\omega) c_{m}^{\dagger}\left(\omega^{\prime}\right)\right\rangle=-\delta_{n m} \delta\left(\omega-\omega^{\prime}\right) f(\omega,-T),
$$

the zero-temperature limit corresponding to a complete population inversion [12]. Higher order expectation values are obtained by pairwise averaging, as one would do for Gaussian variables, after having brought the operators into normal order.

The incoming radiation is in the vacuum state, while the outgoing radiation is collected by a photodetector
[14]. The probability that $n$ photons are counted in a time $t$ is given by $[15,16]$

$$
\begin{gathered}
P(n)=\frac{1}{n !}\left\langle: I^{n} e^{-I}:\right\rangle, \quad I=\int_{0}^{t} d t^{\prime} a^{\text {out } \dagger}\left(t^{\prime}\right) a^{\text {out }}\left(t^{\prime}\right), \\
a^{\text {out }}(t)=(2 \pi)^{-1 / 2} \int_{0}^{\infty} d \omega e^{-\mathrm{i} \omega t} a^{\text {out }}(\omega) .
\end{gathered}
$$

(The colons denote normal ordering.) It is convenient to work with the generating function $F(\xi)=\sum_{p} \kappa_{p} \xi^{p} / p$ ! of the factorial cumulants $\kappa_{p}$ [17],

$$
F(\xi)=\ln \sum_{n=0}^{\infty}(1+\xi)^{n} P(n)=\ln \left\langle: e^{\xi I}:\right\rangle .
$$

To evaluate $F(\xi)$ we substitute Eq. (4) into Eq. (8) and perform the Gaussian averages.

A simple expression results in the long-time regime,

$$
F(\xi)=-t \int_{0}^{\infty} \frac{d \omega}{2 \pi} \ln \left\|\mathbb{1}-\left(\mathbb{1}-S S^{\dagger}\right) \xi f\right\|,
$$

where $\|\cdots\|$ indicates the determinant. Equation (10) is valid when $\omega_{\mathrm{c}} t \gg 1$, with $\omega_{\mathrm{c}}$ the frequency interval within which $S S^{\dagger}$ does not vary appreciably. We have also found a simple expression in the short-time regime,

$$
F(\xi)=-\ln \left\|\mathbb{1}-t \int_{0}^{\infty} \frac{d \omega}{2 \pi}\left(\mathbb{1}-S S^{\dagger}\right) \xi f\right\|,
$$

valid when $\Omega_{\mathrm{c}} t \ll 1$, with $\Omega_{\mathrm{c}}$ the frequency range over which $S S^{\dagger}$ differs appreciably from the unit matrix. (The reciprocal of $\Omega_{\mathrm{c}}$ is the coherence time of the thermal emissions.) The two equations (10) and (11) are the key results of this paper. They reduce the quantum optical problem of the photon statistics to a computation of the scattering matrix of the classical wave equation. That is a major simplification, because the statistical properties of the scattering matrix of a random medium are known from random-matrix theory $[18,19]$.

The long-time limit (10) is particularly simple, as it depends only on the set of eigenvalues $\sigma_{1}, \sigma_{2}, \ldots, \sigma_{N}$ of $S S^{\dagger}$. We call the $\sigma_{n}$ 's "scattering strengths." An additional simplification of the long-time regime is that one can do a frequency-resolved measurement, counting only photons within a narrow frequency interval $\delta \omega$ (with $\omega_{\mathrm{c}} \gg \delta \omega \gg 1 / t$ ). The factorial cumulants are then given by

$$
\kappa_{p}=(p-1) ! \nu f^{p} N^{-1} \sum_{n=1}^{N}\left(1-\sigma_{n}\right)^{p},
$$

where $\nu=N t \delta \omega / 2 \pi$ was defined in the introduction. For comparison with blackbody radiation, we parametrize the variance in terms of the effective number $\nu_{\text {eff }}$ of degrees of freedom [2],

$$
\operatorname{Var}\left[n=\bar{n}\left(1+\bar{n} / \nu_{\mathrm{eff}}\right)\right],
$$


with $\nu_{\text {eff }}=\nu$ for a blackbody. Equation (12) implies

$$
\frac{\nu_{\text {eff }}}{\nu}=\frac{\left[\sum_{n}\left(1-\sigma_{n}\right)\right]^{2}}{N \sum_{n}\left(1-\sigma_{n}\right)^{2}} \leq 1 .
$$

We conclude that the super-Poissonian noise of a random medium corresponds to a blackbody with a reduced number of degrees of freedom. Note that the reduction occurs only for $N>1$.

We now turn to applications of our general formulas to specific random media. We concentrate on the longtime, frequency-resolved regime with $N \gg 1$, leaving the short-time and single-mode regimes, and the case of broadband detection, for future publication [20]. An ensemble of random media has a certain scattering-strength density $\rho(\sigma)$. For $N \gg 1$, sample-to-sample fluctuations are small, so the ensemble average is representative of a single system. We may therefore replace $\sum_{n}$ by $\int d \sigma \rho(\sigma)$ in Eqs. (12) and (14).

As a first example, we compute the thermal radiation from a disordered absorbing slab. The slab is sufficiently thick so that there is no transmission through it, representing a semi-infinite random medium. We define the normalized absorption rate [21] $\gamma=\frac{16}{3} \tau_{\mathrm{s}} / \tau_{\mathrm{a}}$. The scattering-strength density $\rho(\sigma)$ in the regime $\gamma N^{2} \gg 1$ is known [22,23]. It is nonzero in the interval $0<\sigma<$ $\left(1+\frac{1}{4} \gamma\right)^{-1}$, where it equals

$$
\rho(\sigma)=(N / \pi) \sqrt{\gamma}(1-\sigma)^{-2}\left(\sigma^{-1}-1-\frac{1}{4} \gamma\right)^{1 / 2} .
$$

This leads to the effective number of degrees of freedom,

$$
\nu_{\text {eff }} / \nu=4\left[(1+4 / \gamma)^{1 / 4}+(1+4 / \gamma)^{-1 / 4}\right]^{-2},
$$

plotted in Fig. 2, with a mean photocount of

$$
\bar{n}=\frac{1}{2} \nu f \gamma(\sqrt{1+4 / \gamma}-1) .
$$

For strong absorption, $\gamma \gg 1$, we recover the blackbody result $\nu_{\text {eff }}=\nu$, as expected. For weak absorption, $\gamma \ll$ 1 , we find $\nu_{\text {eff }}=2 \nu \sqrt{\gamma}$. In the weak-absorption regime, we can compute the entire distribution $P(n)$ analytically. The result

$$
P(n) \propto\left(\bar{n}^{n} / n !\right)(1+f)^{-n / 2} K_{n-1 / 2}\left(\nu_{\mathrm{eff}} \sqrt{1+f}\right),
$$

with $\bar{n}=\nu f \sqrt{\gamma}$ and $K$ a Bessel function, is Glauber's distribution [15] with a reduced number of degrees of freedom.

Our second example is an optical cavity connected to a photodetector via an $N$-mode waveguide. The cavity modes near frequency $\omega$ are broadened over a frequency range $N \Delta \omega$, much greater than their spacing $\Delta \omega$ if $N \gg$ 1. The cavity should have an irregular shape, or it should contain random scatterers - to ensure chaotic scattering of the radiation. For this system we define the normal-

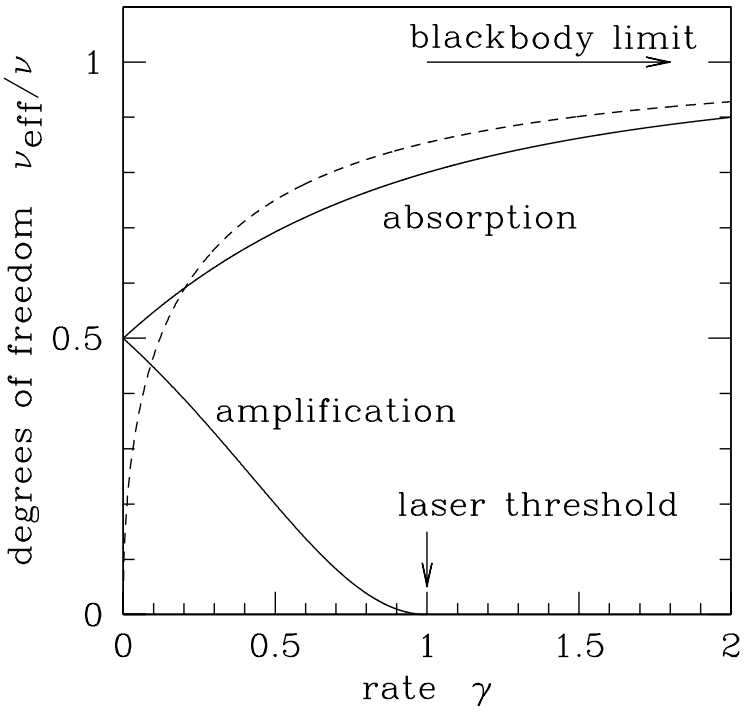

FIG. 2. Effective number of degrees of freedom as a function of normalized absorption or amplification rate. The dashed curve is for the disordered slab, the solid curves are for the chaotic cavity. The amplifying slab would be above the laser threshold for any $\gamma$, so we only plot the case of absorption. For the cavity, both cases of absorption and amplification are shown. The blackbody limit for absorbing systems and the laser threshold for amplifying systems are indicated by arrows.

ized absorption rate as $\gamma=\tau_{\text {dwell }} / \tau_{\mathrm{a}}$, where $\tau_{\text {dwell }} \equiv$ $2 \pi / N \Delta \omega \simeq 1 / \omega_{\mathrm{c}}$ is the mean dwell time of a photon in the cavity without absorption. The scattering-strength density for $N \gg 1$ follows from the general formulas of Ref. [24]. The result has a simple form in the limit $\gamma \ll 1$ of weak absorption,

$$
\rho(\sigma)=(N / 2 \pi)(1-\sigma)^{-2}\left(\sigma-\sigma_{-}\right)^{1 / 2}\left(\sigma_{+}-\sigma\right)^{1 / 2},
$$

for $\sigma_{-}<\sigma<\sigma_{+}$with $\sigma_{ \pm}=1-3 \gamma \pm 2 \gamma \sqrt{2}$. In the opposite limit $\gamma \gg 1$ of strong absorption, $\rho(\sigma)$ is given by the same Eq. (15) as for the disordered slab. We find the effective number of degrees of freedom,

$$
\nu_{\text {eff }} / \nu=(1+\gamma)^{2}\left(\gamma^{2}+2 \gamma+2\right)^{-1},
$$

plotted also in Fig. 2, with a mean photocount of

$$
\bar{n}=\nu f \gamma(1+\gamma)^{-1} .
$$

Again, $\nu_{\text {eff }}=\nu$ for $\gamma \gg 1$. For $\gamma \ll 1$ we now find $\nu_{\text {eff }}=\frac{1}{2} \nu$. It is remarkable that the ratio $\nu_{\text {eff }} / \nu$ for the chaotic cavity remains finite no matter how weak the absorption, while this ratio goes to zero when $\gamma \rightarrow 0$ in the case of the disordered slab.

These two examples concern thermal emission from absorbing systems. As we discussed, our general formulas can also be applied to amplified spontaneous emission, by evaluating the Bose-Einstein function (2) at a negative 
temperature. Complete population inversion corresponds to $f=-1$. A duality relation [25] between absorbing and amplifying systems greatly simplifies the calculation. The dielectric constants $\varepsilon^{\prime} \pm \mathrm{i} \varepsilon^{\prime \prime}$ of dual systems are each other's complex conjugates, so dual systems have the same value of $\tau_{\mathrm{a}}$ and $\gamma$. Their scattering matrices are related by $S_{+}^{\dagger}=S_{-}^{-1}$, hence the scattering strengths $\sigma_{1}, \sigma_{2}, \ldots, \sigma_{N}$ of an amplifying system are the reciprocal of those of the dual absorbing system.

We need to stay below the laser threshold, in order to be in the regime of linear amplification. The semiinfinite medium is above the laser threshold no matter how weak the amplification [22], but the cavity is below threshold as long as $\gamma<1$. We find that $\bar{n}$ and $\nu_{\text {eff }} / \nu$ are given by Eqs. (20) and (21) upon substitution of $\gamma$ by $-\gamma$. In Fig. 2 we compare $\nu_{\text {eff }} / \nu$ for amplifying and absorbing cavities. In the limit $\gamma \rightarrow 0$ the two results coincide, but the $\gamma$ dependence is strikingly different: While $\nu_{\text {eff }} / \nu$ increases with $\gamma$ in the case of absorption, it decreases in the case of amplification-vanishing at the laser threshold. Of course, close to the laser threshold [when $\gamma \geqslant 1-\left(\Omega_{\mathrm{c}} \tau_{\mathrm{dwel1}}\right)^{-1 / 2}$ ] the approximation of a linear amplifier breaks down.

In summary, we have derived a relation between the photocount distribution $P(n)$, in the long-time limit, and the eigenvalues $\sigma_{1}, \sigma_{2}, \ldots, \sigma_{N}$ of the scattering-matrix product $S S^{\dagger}$. The super-Poissonian noise $\operatorname{Var}[n=\bar{n}(1+$ $\left.\left.\bar{n} / \nu_{\text {eff }}\right)\right]$ is that of a blackbody with a reduced number $\nu_{\text {eff }}$ of degrees of freedom. We have computed $\nu_{\text {eff }}$ for several types of random media, in the large- $N$ regime, using results from random-matrix theory. In a weakly absorbing or amplifying chaotic cavity, the ratio $\nu_{\text {eff }} / \nu$ is a universal factor of $1 / 2$-independent of microscopic parameters. In a disordered slab, $\nu_{\text {eff }} / \nu$ vanishes $\propto 1 / \sqrt{\tau_{\mathrm{a}}}$ for small absorption rates $1 / \tau_{\mathrm{a}}$. We have found that $\nu_{\text {eff }} / \nu$ also vanishes on approaching the laser threshold in an amplifying chaotic cavity.

The reduction of $\nu_{\text {eff }}$ amounts to an excess noise of amplified spontaneous emission. Its origin is the presence of a large number $N$ of overlapping cavity modes, and a broad distribution $\rho(\sigma)$ of the corresponding scattering strengths. Overlap of cavity modes is avoided in the usual laser geometry, but it is generic in a random laser. This fundamental difference was pointed out thirty years ago by Letokhov [26], in the paper that pioneered the notion of a "stochastic resonator." Letokhov concludes his paper by surmising that the statistical properties of spontaneous emission would be distinctly different from the usual case. The reduction of the number of degrees of freedom predicted here forms an experimentally accessible signature of this difference.

I have benefited from discussions with P. W. Brouwer, M.P. van Exter, and J.P. Woerdman. This work was supported by the Dutch Science Foundation NWO/FOM.
[1] R. Loudon, The Quantum Theory of Light (Clarendon, Oxford, 1983).

[2] L. Mandel and E. Wolf, Optical Coherence and Quantum Optics (Cambridge University Press, Cambridge, England, 1995).

[3] A. Einstein, Phys. Z. 10, 185 (1909).

[4] J. U. Nöckel and A. D. Stone, Nature (London) 385, 45 (1997).

[5] T. Gruner and D.-G. Welsch, Phys. Rev. A 54, 1661 (1996).

[6] M. Artoni and R. Loudon, Phys. Rev. A 55, 1347 (1997).

[7] Scattering and Localization of Classical Waves in Random Media, edited by P. Sheng (World Scientific, Singapore, 1990).

[8] K. Efetov, Supersymmetry in Disorder and Chaos (Cambridge University Press, Cambridge, England, 1997).

[9] M.L. Mehta, Random Matrices (Academic, New York, 1991).

[10] N. M. Lawandy, R. M. Balachandran, A. S. L. Gomes, and E. Sauvain, Nature (London) 368, 436 (1994).

[11] D. Wiersma and A. Lagendijk, Phys. World 10, 33 (1997).

[12] J. R. Jeffers, N. Imoto, and R. Loudon, Phys. Rev. A 47, 3346 (1993); R. Matloob, R. Loudon, M. Artoni, S. M. Barnett, and J. Jeffers, Phys. Rev. A 55, 1623 (1997).

[13] R. Matloob, R. Loudon, S. M. Barnett, and J. Jeffers, Phys. Rev. A 52, 4823 (1995).

[14] The formulas in the text are for the case of detection efficiency $\alpha=1$ photoelectron per photon. If $\alpha<1$, one should replace $f$ by $\alpha f$.

[15] R. J. Glauber, Phys. Rev. Lett. 10, 84 (1963); in Quantum Optics and Electronics, edited by C. DeWitt, A. Blandin, and C. Cohen-Tannoudji (Gordon and Breach, New York, 1965).

[16] P.L. Kelley and W.H. Kleiner, Phys. Rev. 136, A316 (1964).

[17] The factorial cumulants $\kappa_{p}$ are the cumulants of the factorial moments $\overline{n(n-1) \cdots(n-p+1)}$. For example, $\kappa_{2}=\overline{n(n-1)}-\bar{n}^{2}$.

[18] C. W. J. Beenakker, Rev. Mod. Phys. 69, 731 (1997).

[19] T. Guhr, A. Müller-Groeling, and H. A. Weidenmüller, Phys. Rep. 299, 189 (1998).

[20] C. W. J. Beenakker, in "Diffuse Waves in Complex Media," edited by J.P. Fouque, NATO ASI Series (Kluwer, Dordrecht, to be published).

[21] The coefficient in the definition of $\gamma$ is chosen to facilitate the comparison between slab and cavity.

[22] C. W. J. Beenakker, J. C. J. Paasschens, and P. W. Brouwer, Phys. Rev. Lett. 76, 1368 (1996).

[23] N. A. Bruce and J. T. Chalker, J. Phys. A 29, 3761 (1996).

[24] P. W. Brouwer and C. W. J. Beenakker, J. Math. Phys. 37, 4904 (1996). This reference deals with nonabsorbing cavities. To use its results, we make the mapping between an absorbing cavity with one opening and a nonabsorbing cavity with two openings described by P. W. Brouwer and C. W. J. Beenakker [Phys. Rev. B 55, 4695 (1997)].

[25] J.C. J. Paasschens, T. Sh. Misirpashaev, and C. W. J. Beenakker, Phys. Rev. B 54, 11887 (1996).

[26] V.S. Letokhov, Zh. Eksp. Teor. Fiz. 53, 1442 (1967) [Sov. Phys. JETP 26, 835 (1968)]. 